\title{
BASES PSICONEUROFISIOLÓGICAS DO FENÔMENO PLACEBO-NOCEBO: EVIDÊNCIAS CIENTíFICAS QUE VALORIZAM A HUMANIZAÇÄO DA RELAÇĀO MÉDICO-PACIENTE
}

\author{
Marcus Zulian Teixeira' \\ Trabalho realizado na Faculdade de Medicina da Universidade de São Paulo, São Paulo, SP
}

\author{
*Correspondência: \\ Hospital das Clínicas da \\ FMUSP - Serviço de Clínica \\ Médica Geral. Instituto \\ Central. Prédio dos \\ Ambulatórios. \\ Av. Dr. Enéas de Carvalho \\ Aguiar, $255,4^{\circ}$ andar, bloco \\ 6. CEP 05403-000. São \\ Paulo/ SP. \\ marcus@homeozulian.med.br
}

\begin{abstract}
RESUMO
Aspecto valorizado nos sistemas médicos de todas as épocas, a relação médico-paciente assumiu importância secundária no modelo biomédico vigente, privando o arsenal terapêutico moderno de uma intervenção psiconeurofisiológica capaz de complementar a resolução de muitos distúrbios orgânicos. Com o intuito de resgatar este e outros benefícios, propostas pela humanização da medicina têm surgido nos diversos setores da saúde, restando ao pensamento científico aceitar que este incremento relacional subjetivo possa aumentar a efetividade dos tratamentos convencionais. Funcionando como elemento de sensibilização, os recentes estudos experimentais sobre o fenômeno placebo-nocebo ilustram os posśiveis benefícios ou malefícios que uma relação médico-paciente mais ou menos humanizada pode provocar na evolução das doenças. Unindo as teorias do condicionamento operante inconsciente e da expectativa consciente, as pesquisas sugerem que a postura do médico, permeada por manifestações positivas ou negativas (comentários, sugestões, atitudes etc.), pode exercer influências semethantes no psiquismo dos enfermos, desencadeando respostas neurofisiológicas favoráveis ou desfavoráveis, atuando como instrumento terapêutico ou iatrogênico, respectivamente.
\end{abstract}

UnITERMOs: Efeito placebo. Relações médico-paciente. Humanismo. Humanização da assistência.

\section{INTRODUÇÃO}

Desde os primórdios da humanidade, a prática médica se fundamentou na relação médico-paciente, priorizando a arte de ouvir e examinar cuidadosamente $o$ indivíduo acima de qualquer intervenção terapêutica. Assim sendo, o médico exercia importante influência no psiquismo do enfermo e na evolução das enfermidades, transmitindo através do relacionamento interpessoal a solidariedade, o amparo e a segurança muitas vezes necessários ao despertar da reação vital do organismo. Nestes casos, curava pela simples presença, ou seja, através de efeitos terapêuticos psicológicos ou inespecíficos segundo os parâmetros da medicina científica.

Com o desenvolvimento da biomedicina no século passado, a arte semiológica e a presença do médico cederam espaço aos sofisticados métodos auxiliares de diagnóstico que, associados aos exageros da prescrição medicamentosa pluralista e heroica, desprezaram a relação médico-paciente e os efeitos benéficos deste procedimento terapêutico humanístico, transformando o exercício da Medicina numa prática tecnicista e de alto custo.

No intuito de reverter esta situação, a Comissão da Câmara de Educação Superior do Conselho Nacional de Educação, em 200I, priorizou nas Diretrizes Curriculares Nacionais do Curso de Graduação em Medicinal os aspectos humanísticos, éticos e da integralidade à saúde no perfil do médico generalista recém-formado, estimulando a "interdisciplinaridade em coerência com o eixo de desenvolvimento curricular, buscando integrar as dimensões biológicas, psicológicas, sociais e ambientais".

De forma análoga, procurando resgatar uma relação positiva entre profissionais da saúde e usuários, com nítidos reflexos na qualidade e na eficiência dos serviços prestados, a preocupação com a humanização da saúde passou a ser uma prioridade dos sistemas públicos, com a implantação de propostas nos diversos níveis de atenção: Política Nacional de Humanização (PNH), HumanizaSUS, Programa Nacional de Humanização da Assistência Hospitalar (PNHAH), etc.

Apesar destes movimentos institucionais em defesa do resgate do humanismo na Medicina, o raciocínio médico científico hesita em aceitar que um incremento na relação médico-paciente, aspecto subjetivo e singular perante a diversidade dos poderosos recursos terapêuticos modernos, possa trazer ganhos na eficácia e na eficiência dos tratamentos que o acompanham, desprezando os defensores de semelhante postura.

Estudos experimentais sobre o fenômeno placebo-nocebo começam a transformar este "antigo sermão da Medicina"2, em futura diretriz terapêutica universal, demonstrando como a postura médica, induzindo padrões psicológicos diversos nos pacientes, pode realizar transformações profundas na fisiologia íntima do organismo, que se traduz em efeitos positivos ou negativos na evolução das enfermidades humanas. 


\section{Fenômeno placebo-nocebo baseado em evidências}

Em qualquer tratamento farmacológico, os efeitos terapêuticos relacionam-se a dois tipos de fatores: específicos (dose, duração, via de administração, farmacodinâmica, farmacocinética, interações medicamentosas, etc.) e não específicos (história e evolução natural da doença, regressão à média, aspectos sócioambientais, variabilidade inter e intraindividual, desejo de melhora, expectativas e crenças no tratamento, relação médico-paciente, características nãofarmacológicas do medicamento, etc). O fenômeno placebo-nocebo faz parte destes últimos.

Etimologicamente, o termo placebo se origina do latim placeo, placere, que significa agradar, enquanto o termo nocebo se origina do latim nocere, que significa inflingir dano. De forma generalizada, entende-se efeito ou resposta placebo como a melhoria dos sintomas e/ou funções fisiológicas do organismo em resposta a fatores supostamente inespecíficos e aparentemente inertes (sugestão verbal ou visual, comprimidos inertes, injeção de soro fisiológico, cirurgia fictícia, etc.), sendo atribuível, comumente, ao simbolismo que o tratamento exerce na expectativa positiva do paciente.

Efeito nocebo é um fenômeno oposto ao placebo, em que a antecipação e a expectativa por um resultado negativo podem conduzir à agravação de um sintoma ou doença. Exemplos naturais de efeito nocebo são observados no impacto de diagnósticos negativos e na desconfiança do paciente pela equipe médica ou por um tipo de tratamento, tendo seus mecanismos neurofisiológicos estudados de forma análoga ao efeito placebo ${ }^{3}$.

Segundo de Craen et al. ${ }^{4}$, desde a realização do primeiro ensaio clínico placebo-controlado em 1799, o efeito placebo já trazia importantes ensinamentos para a ciência médica, demonstrando "a maravithosa e poderosa influência das paixões da mente sobre o estado e os distúrbios do corpo". Com a introdução sistemática dos ensaios clínicos randomizados, duplos-cegos e placebos-controlados, considerados o padrão-ouro para avaliar a eficácia dos tratamentos diversos, os relatos frequentes de mudanças clínicas significativas nos grupos placebo conduziram à difusão de que a intervenção placebo pode apresentar efeitos poderosos em diversas condições clínicas.

Algumas metanálises analisaram a resposta placebo (\% de melhora) em doenças isoladas, utilizando ensaios clínicos controlados (ECC) e constatando sua influência em doença de Crohn (32 ECC; 19\%), síndrome da fadiga crônica (29 ECC; 19,6\%) ${ }^{6}$, síndrome do intestino irritável (45 ECC; $40 \%)^{7}$, colite ulcerativa (38 ECC; $\left.26,7 \%\right)^{8}$, depressão maior (75 ECC; $29,7 \%)^{9}$, mania (20 ECC; $\left.31,2 \%\right)^{10}$, enxaqueca (32 ECC; $21 \%)^{\prime \prime}$, etc.

Em 2004, Hrobjartsson e Gotzche publicaram uma revisão sistemática $^{12}$ (I56 ECC; II.737 pacientes) comparando a resposta dos pacientes que receberam ou não intervenção placebo em dez condições clínicas (dor, obesidade, asma, hipertensão, insônia, náusea, depressão, ansiedade, fobia e tabagismo), concluindo que o efeito placebo foi significativo apenas no tratamento da dor e da fobia. Numa re-análise qualitativa destas revisões, Wampold et al. ${ }^{13}$ mostraram que, quando as doenças são suscetíveis ao placebo e o desenho dos estudos é adequado para detectar seus efeitos, o efeito placebo foi robusto. Considerando as ambiguidades das análises, Hunsley e Westmacott ${ }^{14}$ concluem pela existência do fenômeno placebo nos estudos clínicos citados e que "ele não pode ser simples e sumariamente descartado como sem importância ou irrelevante", apesar de ocorrer num grupo reduzido de condições clínicas.

Os diversos fatores envolvidos na relação médico-paciente, do acolhimento ao teor espećfíco das declarações feitas pelo médico, influenciando a expectativa do paciente por uma melhora ou piora do quadro clínico, podem desencadear efeitos significativos no desfecho clínico de qualquer tratamento farmacológico, alterando a atividade de determinadas regiões cerebrais e a liberação de neurotransmissores específicos.

\section{MeCANISMOS MOdULAdORES dO FENÔMENO PLACEBO-NOCEBO}

\section{Condicionamento clássico ou operante inconsciente}

Dentre os mecanismos moduladores do efeito placebo, o condicionamento clássico reivindica que a resposta placebo surge após a exposição repetida do indivíduo a associações de sugestões sensórias neurais (forma e cor dos comprimidos, ambiente do consultório médico, etc.) com intervenções de tratamento efetivas (ex.: morfina no tratamento da dor). Segundo um paradigma estritamente behaviorista (pavloviano), as sugestões sensórias neurais poderiam extrair de forma automática e isolada, após a intervenção placebo, uma resposta semelhante ao tratamento real. Deste modo, o condicionamento estaria relacionado ao fato de que os pacientes são capazes de monitorar rapidamente as flutuações no estado dos órgãos internos pelo feedback sensorial, através da percepção visceral ou somática, e o grau de abrangência desta percepção teria influência proporcional na resposta placebo. De forma análoga, o efeito nocebo seria consequência do condicionamento operante prévio por experiências terapêuticas negativas ${ }^{15-16}$.

Estudando o condicionamento operante no sistema imunológico, Olness e Ader $^{17}$ condicionaram crianças portadoras de lúpus eritematoso com a administração de ciclofosfamida associada a estímulos gustativos e olfativos, conseguindo imunossupressão placebo durante o curso de doze meses de administração isolada dos estímulos gustativos e olfatórios. Goebel et al. ${ }^{18}$, após a indução de imunossupressão condicionada por solução de sacarina com ciclosporina $A$, observaram imunossupressão placebo (alterações na IL-2 e INF-g) após a administração isolada de solução de sacarina. Em relação ao sistema cardiovascular, Benedetti et al..$^{19}$ mostraram que o condicionamento prévio pela repetição de doses analgésicas de buprenorfina, indutoras de hipoventilação moderada como efeito colateral, permitiu que a posterior analgesia placebo mimetizasse a mesma depressão respiratória. Esta resposta placebo respiratória colateral foi totalmente bloqueada pela naloxona (antagonista opioide), indicando ter sido mediada pelos mesmos opioides endógenos da resposta placebo analgésica principal. No sistema endócrino, após o pré-condicionamento com sumatripan, um agonista serotoninérgico que estimula a secreção do hormônio do crescimento $(G H)$ e inibe a secreção do cortisol, a administração do placebo estimulou os mesmos efeitos da droga ${ }^{20}$.

\section{Expectativa consciente}

Um importante mecanismo psicológico modulador do efeito placebo é a expectativa consciente dos pacientes nas perspectivas de 
melhoras clínicas, que pode ser incrementada pelas sugestões verbais que acompanham o tratamento placebo.

Um modelo experimental peculiar tem avaliado o impacto clínico das expectativas positiva e negativa isoladamente, revelando ou ocultando ao paciente a administração ou a suspensão do tratamento mais bem indicado (open-hidden paradigm). Diversos estudos têm mostrado que um mesmo tratamento mostra-se mais efetivo quando é revelado (open) do que quando é ocultado (hidden) ao paciente, indicando que a expectativa positiva (efeito psicológico) desempenha um papel crucial no desfecho do tratamento. Considerando que o desfecho clínico secundário a um tratamento não revelado (hidden) representa o efeito específico do tratamento em si, livre de qualquer contaminação psicológica, o resultado de um tratamento revelado (open) representa a somatória dos efeitos específico e psicológico. A diferença entre os efeitos dos tratamentos revelado e ocultado tem sido considerada como a representação do componente placebo, embora nenhum placebo tenha sido administrado ${ }^{21-22}$. De forma análoga, a expectativa negativa foi avaliada com a revelação ou a ocultação da suspensão do tratamento indicado ${ }^{23}$, mostrando que o grupo que sabia da interrupção apresentou piora dos sintomas de forma mais intensa e antecipada ao outro grupo. A diferença entre os efeitos da suspensão do tratamento nos dois grupos representaria o efeito nocebo.

\section{Expectativa consciente + condicionamento operante}

Apesar dos defensores incondicionais de um ou outro mecanismo, expectativa consciente e condicionamento operante inconsciente são complementares. Estudos indicam que tanto a expectativa consciente quanto o condicionamento operante inconsciente são mecanismos coadjuvantes na modulação da analgesia placebo, um amplificando a resposta do outro.

Amanzio e Benedetti ${ }^{23}$ estudaram a interação entre estes mecanismos em indivíduos no pós-operatório de cirurgia odontológica, segundo quatro tipos de intervenções: "expectativa" (infusão salina com sugestão verbal de que era um poderoso analgésico), "condicionamento" (dois dias de condicionamento em que morfina foi associada com infusão salina, dizendo aos sujeitos que era um antibiótico para "limpar o sangue"), "expectativa + condicionamento" e "nenhum tratamento" (controle). Comparados ao grupo controle, os grupos "expectativa" e "condicionamento" mostraram cada qual um pequeno, mas significativo aumento na tolerância à dor, enquanto o grupo que recebeu "expectativa + condicionamento" apresentou tolerância à dor duas vezes maior que os grupos das intervenções isoladas.

$\mathrm{Na}$ proposta de um modelo que adiciona o desejo à expectativa consciente (modelo desejo-expectativa) ${ }^{24}$, estudos sugerem que a resposta placebo seria co-determinada por metas, desejos, expectativas e os sentimentos emocionais que as acompanham. Acrescentando o condicionamento operante a este modelo, experimentos mostram que a percepção somática das mudanças internas facilita a retroalimentação destes fatores ao longo do tempo, selecionando sentimentos emocionais mais positivos (ou menos negativos) na perspectiva de evitar experiências aversivas ou obter experiências desejáveis $^{15}$. De forma análoga, uma dinâmica mental negativista atuaria em sentido oposto, estimulando a resposta nocebo.

\section{MECANISMOS NEUROFISIOLÓgICOS MEDIADORES DO FENÔMENO PLACEBO-NOCEBO}

\section{Fenômenos dolorosos}

A resposta placebo analgésica tem como mediadores os peptídeos opioides endógenos cerebrais (endorfinas), que atuam nos mesmos sítios dos receptores dos analgésicos opioides exógenos (morfina) distribuídos em regiões cerebrais específicas (tronco encefálico, tálamo e medula espinhal), atingindo grandes concentrações no líquido espinhal. Esta hipótese foi confirmada por uma série de experimentos que evidenciaram o bloqueio da resposta placebo analgésica pela infusão de naloxona, antagonista dos receptores opioides ${ }^{25}$.

Dentre os mecanismos moduladores da analgesia placebo, postulase que a expectativa pela diminuição da dor deve estimular os opioides endógenos, enquanto o condicionamento operante deve ativar subsistemas específicos insensíveis à naloxona. O efeito placebo analgésico ainda pode ser mediado pela estimulação dos circuitos dopaminérgicos, e pela inibição dos circuitos prostaglandina-dependentes e colecistoquinase-dependentes.

Utilizando um modelo experimental de dor induzida pelo calor, os efeitos neurofisiológicos das analgesias opioide e placebo foram analisados em nove voluntários sadios através de tomografia por emissão de positrons (PET), comparando-se as áreas cerebrais ativadas. Tanto o placebo quanto o agonista opioide (remifentanil) ativaram áreas cerebrais espećíicas, sugerindo uma relação direta entre ambos tipos de analgesia: córtex pré-frontal medial (córtex cingular anterior ou cíngulo anterior), córtex pré-frontal orbitofrontal (córtex somato-sensorial) e sistema opióide do tronco encefálico (substância cinzenta periaquedutal ou SCP ou PAG). No grupo da analgesia placebo, o aumento da atividade no cíngulo anterior ocorreu somente nos pacientes classificados independentemente como placebo-respondedores ${ }^{26}$.

Em dois experimentos com ressonância nuclear magnética funcional (RNMf), Wager et al. ${ }^{27}$ observaram diminuição da dor com a aplicação de um creme placebo nos braços de pacientes submetidos a choques elétricos, que coincidiu com o aumento da atividade cerebral no córtex pré-frontal orbitofrontal, córtex pré-frontal dorsolateral, córtex pré-frontal medial e SCP (regiões relacionadas à modulação emocional da expectativa e da antecipação do fenômeno doloroso), e diminuição da atividade cerebral no tálamo, córtex insular anterior e córtex cingular anterior (regiões relacionadas à dor).

De forma análoga, Price et al..$^{28}$ mapearam a atividade cerebral (RNMf) logo após a administração de analgesia placebo em pacientes com síndrome do intestino irritável, observando o efeito da sugestão verbal (expectativa positiva) na diminuição da atividade neural das áreas cerebrais relacionadas à dor (tálamo, córtex insular anterior e córtex cingular anterior), acompanhada pelo aumento da atividade cerebral nas áreas responsáveis pela modulação emocional da dor (córtex cingular anterior, amígdala lateral e SCP).

Aprofundando o entendimento do circuito neural da analgesia placebo, Fricchione e Stefano ${ }^{29}$ salientam que caberia ao córtex préfrontal dorsolateral a propriedade de manter e refinar representações internas das expectativas, ativando posteriormente as demais regiões cerebrais. Expectativas positivas em relação à analgesia estimulariam 
então o córtex pré-frontal dorsolateral que ativaria simultaneamente o córtex pré-frontal medial, o córtex pré-frontal orbitofrontal (antecipação da dor) e o sistema opioide do tronco encefálico (SCP), áreas responsáveis pela modulação da dor emocional, desencadeando a analgesia.

Em relação ao efeito nocebo hiperálgico, outros estudos experimentais têm mostrado que a percepção da intensidade do estímulo doloroso é amplificada após uma expectativa (sugestão verbal) negativa de aumento da dor, e que diversas regiões cerebrais (córtex pré-frontal orbitofrontal, córtex cingulado anterior e córtex insular anterior) apresentam atividade aumentada durante a antecipação da dor. Estas alterações cerebrais mostraram-se opostas às encontradas durante a indução de uma expectativa positiva de diminuição da dor ${ }^{30}$.

Keltner et al. ${ }^{31}$ encontraram que o nível de intensidade da expectativa dolorosa térmica (efeito nocebo hiperálgico) altera a intensidade da dor percebida, juntamente à ativação de regiões do cérebro visualizadas na RNMf: expectativa negativa secundária a um estímulo visual nocivo de alta intensidade ativou distintas regiões do cérebro (tálamo, córtex insular anterior, córtex cingular anterior, córtex somato-sensorial, amígdala, estriado ventral e os núcleos cuneiformes no tronco encefálico) em intensidade superior a um estímulo visual nocivo de baixa intensidade.

\section{Doença de Parkinson}

Em 2002, Pollo et al..$^{32}$ estudaram a influência das expectativas positiva e negativa na melhora motora em pacientes portadores de doença de Parkinson que tinham implantado eletrodos no núcleo subtalâmico (NST) para estimulação cerebral profunda, tratamento paliativo para os sintomas motores da doença (tremor, rigidez muscular e lentificação dos movimentos). Empregando um analisador de movimentos, avaliaram o efeito da estimulação subtalâmica sobre a velocidade do movimento da mão direita, observando maior rapidez dos movimentos quando a estimulação foi acompanhada de expectativa positiva de melhora (efeito placebo) e lentificação com expectativa negativa (efeito nocebo). Vale ressaltar que estes efeitos opostos ocorreram em minutos, indicando que a expectativa induz mudanças neurais rapidamente. Confirmando estes resultados, outros estudos indicaram que, dentre os sintomas motores, a bradicinesia é mais sensível à sugestão verbal do que o tremor e a rigidez, desconhecendo-se seu mecanismo neurofisiológico 3,20.

Utilizando PET com ("C)raclopride, um antagonista dos receptores dopaminérgicos, de la Fuente-Fernandez et al., ${ }^{33}$ estudaram o efeito da intervenção placebo em seis pacientes portadores de doença de Parkinson. Em resposta às injeções subcutâneas de solução salina, foram liberadas quantidades significativas de DA endógena, presumivelmente de sinapses dos núcleos caudado e putamen (estriado dorsal), desalojando o raclopride marcado dos receptores dopaminérgicos da região. Esta liberação de DA foi maior em pacientes que perceberam melhoras com o placebo (placebos-respondedores) do que naqueles que não perceberam. $\mathrm{O}$ aumento da DA endógena liberada no estriado dorsal sugere que o efeito placebo em doença de Parkinson é mediado pela ativação dos depósitos de DA nigrostriatal com subsequentes efeitos sinápticos, embora este sistema esteja danificado no Parkinson. O placebo induziu uma mudança na concentração do antagonista raclopride comparável ao de doses terapêuticas de levodopa. Os autores acreditam que a expectativa relacionada à antecipação do benefício terapêutico e acompanhada pela liberação de DA endógena pode ser um fenômeno comum ao efeito placebo, em qualquer distúrbio clínico placebo-responsivo.

\section{Depressão}

A magnitude da resposta placebo no tratamento da depressão maior foi avaliada em revisão sistemática de 75 estudos placeboscontrolados 9 , mostrando que o grupo placebo apresentou um efeito terapêutico médio de $29,7 \%$ e o grupo ativo de $50,1 \%$. Chegando a valores semelhantes, Sysko e Walsh ${ }^{10}$ avaliaram o efeito placebo no tratamento da mania bipolar (lítio e risperidona), encontrando uma resposta terapêutica média de $31,2 \%$ no grupo placebo e de 55,9\% no grupo ativo.

Em recente metanálise que avaliou a eficácia clínica dos antidepressivos inibidores seletivos da recaptação de serotonina (ISRS) no tratamento da depressão maior, Kirsch et al. ${ }^{34}$ concluíram não haver diferença significativa frente ao placebo nos pacientes com depressão moderada e apenas "uma pequena e insignificante diferença nos pacientes com depressão muito severa", os quais apresentam diminuição da resposta placebo.

Com o intuito de distinguir as mudanças na atividade cerebral que o tratamento placebo produziria em pacientes depressivos, Leuchter et al. ${ }^{35}$ conduziram um ensaio clínico com $5 \mathrm{I}$ indivíduos portadores de depressão maior que receberam antidepressivos ou placebo durante nove semanas, sendo então classificados como "respondedores ao medicamento", "respondedores ao placebo", "não-respondedores ao medicamento" e "não-respondedores ao placebo". Utilizando a eletroencefalografia quantitativa (EEGQ) como marcador da atividade cerebral pré e pós-tratamentos, observaram que enquanto o grupo pré-tratamento não apresentou diferenças eletroencefalográficas quantitativas, os "respondedores ao placebo" mostraram um aumento significativo da cordance no córtex pré-frontal, que é uma medida da atividade eletroencefalográfica que apresenta forte correlação com a perfusão cerebral quantificada pela PET. Esta alteração foi observada precocemente no curso do tratamento e não foi vista no grupo dos "não-respondedores" nem no grupo dos "respondedores ao medicamento", que mostrou diminuição da cordance pré-frontal.

Mayberg et al. ${ }^{36}$, empregando PET no estudo da resposta placebo no metabolismo regional da glicose cerebral em 17 pacientes masculinos com depressão maior, conduziram um ensaio clínico duplo-cego e placebo-controlado em que os efeitos tomográficos da fluoxetina foram comparados com os do placebo. A remissão dos sintomas foi observada em oito (Quatro de cada grupo) dos 15 indivíduos que completaram o estudo. Observou-se que a resposta placebo estava associada ao aumento do metabolismo da glicose no córtex pré-frontal, cíngulo anterior, córtex parietal, insula posterior e cíngulo posterior, além da diminuição do metabolismo no cíngulo subgenual, parahipocampo e tálamo. Os que responderam à fluoxetina mostraram um padrão metabólico similar, sugerindo um possível envolvimento da serotonina nos efeitos antidepressivos da indução placebo. Petrovic et al. ${ }^{26}$, em estudo anteriormente citado, observaram mudanças semeIhantes no metabolismo da glicose cerebral. 


\section{Integração dos mecanismos psiconeurofisiológicos do fenô- meno placebo-nocebo}

Segundo Benedetti et al. ${ }^{3}$, existem evidências suficientes para afirmar que a expectativa positiva ativaria os opioides endógenos e as interconexões moduladoras da dor, diminuindo a transmissão nos trajetos dolorosos, induzindo a liberação de dopamina no estriado e afetando a atividade de neurônios únicos no NST. Na depressão, existe alguma evidência experimental que diferentes regiões cerebrais relacionadas à serotonina estariam envolvidas na resposta placebo. Por outro lado, evidências experimentais recentes indicam que sugestões verbais negativas induzem ansiedade antecipatória sobre o provável aumento da dor, ativando o sistema colecistoquinérgico facilitador da transmissão dolorosa e diminuindo a atividade dos opioides endógenos, promovendo hiperalgesia nocebo. O efeito nocebo também mostrou afetar experimentalmente outras condições clínicas, como a doença de Parkinson.

Baseados no fato de que a expectativa pode estar relacionada a uma ativação tônica dos neurônios dopaminérgicos, que se projetam para o estriado dorsal, estriado ventral e córtex pré-frontal, de la Fuente-Fernandez et al. ${ }^{16}$ propuseram o seguinte mecanismo psiconeurofisiológico para explicar o efeito placebo: quando qualquer tipo de intervenção placebo cria uma expectativa positiva de recompensa, neurônios do córtex pré-frontal são ativados pela probabilidade de ocorrência da mesma; por sua vez, estas células enviam impulsos glutamatérgicos excitatórios diretos e impulsos gabaérgicos inibitórios indiretos aos neurônios dopaminérgicos distribuídos ao longo do corpo, estando na combinação destes sinais o requisito para sua ativação tônica. Além disso, neurônios do córtex pré-frontal, estriado dorsal e estriado ventral podem exibir ativações tônicas durante a expectativa de recompensa. Existe também a ativação dopaminérgica fásica, que acontece após a recompensa efetiva, sendo muito forte quando esta surge inesperadamente.

Unindo ambos mecanismos, Fricchione e Stefano ${ }^{29}$ propõe que a convicção em uma expectativa positiva pode afetar uma mudança na atividade do córtex pré-frontal, cuja retroalimentação estimula o fluxo de dopamina do fascículo medial para o tegumento ventral. Receptores no estriado dorsal (núcleos caudado e putamen), no estriado ventral (núcleos da base) e no córtex pré-frontal medial, orbitofrontal e dorsolateral registrarão esta mudança tônica de dopamina. Ao mesmo tempo, a ativação do córtex pré-frontal por expectativa positiva pode estimular também o sistema opioide do tronco cerebral (SCP).

Acrescentando a reaçãa ao estresse e o óxido nítrico $(\mathrm{NO})$ ao mecanismo de resposta placebo, os autores explicam-na através do seguinte modelo psiconeurofisiológico: iniciando pela convicção de melhora gerada no córtex pré-frontal pelo estímulo sensório talâmico, a mensagem de expectativa positiva é transmitida por tratos corticolímbico-estriatais para os núcleos da base, por onde vários mediadores podem trabalhar via NO para liberar o fluxo de dopamina para o movimento, a recompensa e os circuitos de motivação. Desta forma, o poder da convicção e da expectativa consciente positiva ao qual o efeito placebo está relacionado alteraria o padrão neuroquímico em áreas chaves do cérebro responsáveis pelo movimento (corpo estriado), prazer (núcleos da base) e dor física ou psicológica (cíngulo anterior), desencadeando uma resposta placebo evidente em doença de Parkinson, transtornos depressivos e distúrbios dolorosos, respectivamente. Entretanto, o sistema dopaminérgico mesolímbicomesocortical também sofre controle superior dos eixos de resposta ao estresse (hipotálamo-pituitária-adrenal e amígdala lateral-locus coeruleus), sugerindo que a convicção e a expectativa positiva podem modificar a resposta ao estresse e conduzir o efeito placebo para muitos distúrbios psicossomáticos como hipertensão, angina, doença intestinal inflamatória e asma, dentre outras ${ }^{29}$.

\section{Conclusão}

Em vista do crescente número de pesquisas que atestam a existência e a dinâmica do fenômeno placebo-nocebo, a classe médica começa a ter acesso a evidências científicas que justificam a incorporaçãa de atitudes humanizadas na relação com seus pacientes, a fim de incrementar a eficácia dos tratamentos convencionais com esta terapêutica psiconeurofisiológica.

Dedicando uma parte do tempo da consulta técnica e objetiva à escuta solidária da subjetividade psicosocioambiental que permeia a individualidade humana, o médico saberá como estimular as expectativas conscientes positivas dos pacientes no sentido curativo dos distúrbios que os acompanham (efeito placebo), podendo acrescentar aos modernos protocolos terapêuticos um incremento de melhora exclúido das estatísticas vigentes. Por outro lado, dispensará maior vigilância às palavras, gestos e atitudes permeados por sentimentos destituídos dos valores ético-humanistas que, ao induzirem expectativas negativas nos pacientes (efeito nocebo), poderão despertar efeitos iatrogênicos contrários ao ideal de cura dos tratamentos propostos.

No entanto, pelos resultados apresentados, não temos como inferir uma suposta especificidade e magnitude do fenômeno placebo-nocebo de forma generalizada. Dependendo do tipo de sintoma ou doença, da informação transmitida ao paciente, da expectativa (associada ou não à sugestão verbal), das experiências prévias do paciente com as diversas situações e tratamentos (condicionamento operante), da sensibilidade individual etc., os efeitos da intervenção placebo-nocebo podem diferir na magnitude e na especificidade, envolvendo diferentes mecanismos psicológicos e suas respectivas áreas cerebrais.

\section{SUMMARY}

Psiconeurophysiologic bases OF the PLACEBO-NOCEBo PHENOMENON: SCIENTIFIC EVIDENCES THAT VALUE THE HUMANIZATION OF THE DOCTOR-PATIENT RELATIONSHIP

Aspect quite valued in the medical systems of all of the times, the doctor-patient relationship assumed secondary importance in the current biomedical model, depriving the modern therapeutic arsenal of a psyconeurophisiological intervention capable to complement the resolution of many organic disturbances. With the intention of rescuing this and other benefits, countless proposed by the humanization of the medicine have been appearing in the several sections of the health, remaining to scientific thought accept that this subjective relational increment can increase the effectiveness of the conventional treatments. Working as sensitization element, the recent experimental studies illustrate the possible benefits or harms that a doctor-patient relationship more or less humanized can provoke in the evolution of the 
diseases. Uniting the theories of classical conditioning and conscious expectation, the researches suggest that the doctor's posture, permeated by positive or negative manifestations (comments, suggestions, attitudes etc.), it can exercise similar influences in the patients' psyche, unchaining favorable or unfavorable neurophysiologic answers, acting as therapeutic or iatrogenic instrument, respectively. [Rev Assoc Med Bras 2009; 55(I): 13-8]

KEY wORDS: Placebo effect. Physician-patient relationship. Humanism. Humanization of assistance.

\section{REFERÊNCIAS}

I. Diretrizes Curriculares Nacionais do Curso de Graduação em Medicina. Conselho Nacional de Educação. Câmara de Educação Superior. Resolução CNE/CES n 4, de 07 de novembro de 2001 .

2. Cho HJ. Reviving the old sermon of medicine with the placebo effect. Rev Bras Psiquiatr. 2005;27:336-40.

3. Benedetti F, Lanotte M, Colloca L. When words are painful: unraveling the mechanisms of the nocebo effect. Neuroscience. 2007;147:260-71.

4. de Craen AJ, Kaptchuk TJ, Tijssen JG, Kleijnen J. Placebos and placebo effects in medicine: historical overview. J R Soc Med. 1999;92:5। I-5.

5. Su C, Lichtenstein GR, Krok K, Brensinger CM, Lewis JD. A meta-analysis of the placebo rates of remission and response in clinical trials of active Crohns disease. Gastroenterology. 2004; 126: 1257-69.

6. Cho HJ, Hotopf M, Wessely S. The placebo response in the treatment of chronic fatigue syndrome: a systematic review and meta-analysis. Psychosom Med. 2005;67:30 I- I3.

7. Patel SM, Stason WB, Legedza A, Ock SM, Kaptchuk TJ, Conboy L, et al. The placebo effect in irritable bowel syndrome trials: a meta-analysis. Neurogastroenterol Motil. 2005; 17:332-40.

8. Inyckyj A, Greenberg H, Bernstein CN. Quantification of the placebo response in ulcerative colitis. Gastroenterology. 1997; I I 2: 1854-8.

9. Walsh BT, Seidman SN, Sysko R, Gould M. Placebo response in studies of major depression: variable, substantial, and growing. JAMA. 2002;287: 1 840-7.

10. Sysko R, Walsh BT. A systematic review of placebo response in studies of bipolar mania. J Clin Psychiatry. 2007;68:1213-7.

I I. Macedo A, Baños JE, Farré M. Placebo response in the prophylaxis of migraine: a meta-analysis. Eur J Pain. 2008; 12:68-75.

12. Hrobjartsson A, Gotzsche PC. Is the placebo powerless? Update of a systematic review with 52 new randomized trials comparing placebo with no treatment. J Intern Med. 2004;256:91- 100.

13. Wampold BE, Minami T, Tierney SC, Baskin TW, Bhati KS. The placebo is powerful: estimating placebo effects in medicine and psychotherapy from randomized clinical trials. J Clin Psychol. 2005;63:373-7.

14. Hunsley J, Westmacott R. Interpreting the magnitude of the placebo effect: mountain or Molehill? J Clin Psychol. 2007;63:391-9.

15. Geers AL, Helfer SG, Weiland PE, Kosbab K. Expectations and placebo response: a laboratory investigation into the role of somatic focus. J Behav Med. 2006;29:171-8.

16. de la Fuente-Fernandez R, Schulzer M, Stoessl AJ. Placebo mechanisms and reward circuitry: clues from Parkinson's disease. Biol Psychiatry. 2004;56:67-7I.

17. Olness K, Ader R. Conditioning as an adjunct in the pharmacotherapy of lupus erythematosus. J Dev Behav Pediatr. 1992; 13: I24-5.

18. Goebel MU, Trebst AE, Steiner J, Xie YF, Exton MS, Frede S, et al. Behavioral conditioning of immunosuppression is possible in humans. FASEB J. 2002; 16: 1869-73.
19. Benedetti F, Amanzio M, Baldi S, Casadio C, Maggi G. Inducing placebo respiratory depressant responses in humans via opioid receptors. Eur J Neurosci. 1999; | 1:625-31.

20. Benedetti F, Pollo A, Lopiano L, Lanotte M, Vighetti S, Rainero I. Conscious expectation and unconscious conditioning in analgesic, motor, and hormonal placebo/nocebo responses. J Neurosci. 2003;23:43। 5-23.

21. Amanzio M, Pollo A, Maggi G, Benedetti F. Response variability to analgesics: a role for non-specific activation of endogenous opioids. Pain 2001;90:205-I5.

22. Colloca L, Lopiano L, Lanotte M, Benedetti F. Overt versus covert treatment for pain, anxiety, and Parkinson's disease. Lancet Neurol. 2004:3:679-84.

23. Amanzio M, Benedetti F. Neuropharmacological dissection of placebo analgesia: expectation-activated opioid systems versus conditioningactivated subsystems. J Neurosci. 1999;484-94.

24. Vase L, Robinson ME, Verne GN, Price DD. Increased placebo analgesia over time in irritable bowel syndrome (IBS) patients is associated with desire and expectation but not endogenous opioid mechanisms. Pain. 2005; 1 1 5:338-47.

25. Sauro MD, Greenberg RP. Endogenous opiates and the placebo effect. A meta-analytic review. J Psychosom Res. 2005;58: I I 5-20.

26. Petrovic $P$, Kalso E, Petersson KM, Ingvar M. Placebo and opioid analgesia: imaging a shared neural network. Science. 2002;295: 1737-40.

27. Wager TD, Rilling JK, Smith EE, Sokolic A, Casey KL, Davidson RJ, et al. Placebo-induced changes in $\mathrm{FMRI}$ in the anticipation and experience of pain. Science. 2004;303:1 162-7.

28. Price DD, Craggs J, Verne GN, Perlstein WM, Robinson ME. Placebo analgesia is accompanied by large reductions in pain-related brain activity in irritable bowel syndrome patients. Pain. 2007;127:63-72.

29. Fricchione G, Stefano GB. Placebo neural systems: nitric oxide, morphine and the dopamine brain reward and motivation circutries. Med Sci Monit. 2005; I I:MS54-65.

30. Koyama T, McHaffie JG, Laurienti PJ, Coghill RC. The subjective experience of pain: where expectations become reality. Proc Natl Acad Sci USA. 2005; 102: 12950-5.

31. Keltner JR, Furst A, Fan C, Redfern R, Inglis B, Fields HL. Isolating the modulatory effect of expectation on pain transmission: a functional magnetic resonance imaging study. J Neurosci. 2006;26:4437-43.

32. Pollo A, Torre E, Lopiano L, Rizzone M, Lanotte M, Cavanna A. Expectation modulates the response to subthalamic nucleus stimulation in Parkinsonian patients. Neuroreport. 2002; I3: |383-6.

33. de la Fuente-Fernandez R, Ruth TJ, Sossi V, Schulzer M, Calne DB, Stoessl AJ. Expectation and dopamine release: mechanisms of the placebo effect in Parkinson's disease. Science. 2001;293:1 I64-6.

34. Kirsch I, Deacon BJ, Huedo-Medina TB, Scoboria A, Moore TJ, Johnson BT. Initial severity and antidepressant benefits: a meta-analysis of data submitted to the Food and Drug Administration. PLoS Med 2008;5(2):e45.

35. Leuchter AF, Cook IA, Witte EA, Morgan M, Abrams M. Changes in brain function of depressed subjects during treatment with placebo. Am J Psychiatry. 2002; 159: I 22-9.

36. Mayberg HS, Silva JA, Brannan SK, Tekell JL, Mahurin RK, McGinnis S et al. The functional neuroanatomy of the placebo effect. Am J Psychiatry. 2002; | 59:728-37.

Artigo recebido: 22/08/08

Aceito para publicação: 13/10/08 